1. Hurt RD, Sachs DPL, Glover ED, Offord KP, Johnston JA, Dale LC, et al. A comparison of sustained-release bupropion and placebo for smoking cessation. N Engl J Med 1997; 337: 1195-1202.

2. Glasman AH, Covey LS. Smoking and affective disorder. Am J Health Behav 1996; 20: 279-285.

3. Joremby DE, Leischow SJ, Nides MA, Rennard SI, et al. A controled trial of sustained-release bupropion, a nicotine patch, or both for smoking cessation. N Engl J med 1999; 340: 685-691.

4. Clifford DB, Rutherford JL, Hicks FG, Zorumski CF. Acute effects of antidepressants on hipocampal seizures. Ann Neurol 1985; 18: 692-7.

5. E. Cárdenas, E. Caballero, J. Honorato. Nuevos Medicamentos: Bupropion. Rev Med Univ Navarra 2001; 45 (3): 51-55.

6. Goodale E, Ascher J, Batey S. Cytochrome P450 metabolism of bupropion (abstract). J Am Geriatr Soc 1999; 47: S88.

7. Ascher JA, Cole JO, Colin JN, Feghner JP, et al. Bupropion: a review of its mechanism of antidepressant activity. J Clin Psychyatry 1995; 56: 395-401.

8. Kwan AL, Meiners AP, Von Grootheest AC, Lakkerker JF. Risk of convulsions due to the use of bupropion as an aid for smoking cessation. Need Tijdsch Geneeskd 2001 ; 145(6): 277-8.

9. Johnston JA, Lineberry CG, Ascher JA, Davidson J, et al. A 102-center prospective study of seizure in association with bupropion. J Clin Psychiatry 1993; 54: 202.

10. Minie I, McMcGurk C, McClintock G, Robinson J. Seizures after Bupropion overdose. Lancet 2001; 357 (9268): 1624.

\section{Púrpura trombótica trombocitopénica como debut de un cuadro neoplásico}

\section{Sr. Director:}

Aunque es bien conocida la asociación de anemia hemolítica microangiopática y de coagulación intravascular diseminada (CID) como complicaciones de carcinomas diseminados, la asociación con la púrpura trombótica trombocitopénica (PTT) es mucho más inusual aunque está descrita en la literatura.

Presentamos el caso de una mujer de 34 años, fumadora importante, sin antecedentes de interés salvo el diagnóstico de embolismo pulmonar hacía un mes, y con tratamiento anticoagulante desde entonces, que reingresa por deterioro del estado general, disnea progresiva siendo de reposo a su llegada y pérdida de diez kilos de peso en los últimos seis meses. A la exploración física presentaba un regular estado general, febrícula y leve taquipnea. Se palpaba adenopatía supraclavicular derecha de dos centímetros y axilares homolaterales de menor tamaño, siendo el resto de la exploración anodina. En la analítica presentaba: $\mathrm{Hb}: 8,4$, plaquetas: 60.000 , LDH: 1561 , siendo el resto de los parámetros normales. Gasometría arterial: $\mathrm{pO}_{2}$ : 66, $\mathrm{pCO}_{2}: 24, \mathrm{PH}: 7,4$. RX de tórax: agrandamiento hiliar bilateral y ensanchamiento mediastínico. TAC tóraco-abdominal: adenopatías mediastínicas, axilares, periportales y retroperitoneales múltiples. En el ecocardigrama se objetivó hipertensión pulmonar ligera. Al presentar nuevamente insuficiencia respiratoria, se sospechó inicialmente nuevo episodio de tromboembolismo, que coincidiendo con la presencia de adenopatías y alteraciones hematológicas hizo pensar como primera posibilidad diagnóstica de base en un linfoma. Se realizó Biopsia de Médula ósea que mostró marcada hiperplasia de la serie roja sin evidencia de infiltración tumoral. CEA: 351,2; Ca 15,3: 129, resto de marcadores normales. Se solicitó al Servicio de Cirugía biopsia del ganglio supraclavicular para intentar filiar histológicamente el cuadro. A las 72 horas, presentó fiebre, empeoramiento de su disnea, pérdida de visión de ojo derecho, parestesias y paresia de extremidad superior derecha. Estaba ictérica, taquicárdica y taquipneica y con equimosis cutáneas múltiples. Hb: 6, plaquetas: 43.000, bilirrubina de 4,7 de predominio indirecto, LDH: 2005, haptoglobina: 6, resto de funciones hepática y renales normales. Con la sospecha de hemólisis se realizaron un test de Coombs directo e indirecto que fueron negativos y un frotis en sangre periférica que mostró abundantes equistocitos. Con estos datos de anemia hemolítica microangiopática, se inició tratamiento con esteroides e infusión de plasma fresco. El empeoramiento de su disnea y la existencia de hipertensión pulmonar nos llevó a realizar una arteriografía pulmonar, por sospecha de nuevo embolismo, que mostró un déficit importante de flujo vascular periférico, manteniendo la vascularización intacta en los troncos principales. La paciente se deterioró gravemente en las horas siguientes con estado confusional. El resultado anatomopatológico de la biopsia fue de metástasis de adenocarcinoma de células en anillo de sello, destacando el origen gástrico como primera posibilidad. La paciente falleció en las dos horas siguientes. El diagnóstico fue de adenocarcinoma metastásico de probable origen gástrico y púrpura trombótica trombocitopénica asociada.

La PTT (1) es un síndrome desencadenado por la oclusión diseminada de la microcirculación y que clínicamente se caracteriza por anemia hemolítica, trombocitopénia, síntomas neurológicos, fiebre y disfunción renal. Otras manifestaciones incluyen cuadros hemorrágicos, malestar general, naúseas y vómitos, disnea y arritmias. El diagnóstico diferencial $(2,3)$ debe hacerse fundamentalmente con la CID, la cual cursa con alteraciones de la coagulación y con el síndrome hemolítico urémico, que corresponde con la afectación local renal de dicho proceso microangiopático. En cuanto al desencadenante etiológico (4) en general afecta a personas sana, cuando es secundaria se ha relacionado con el embarazo, infecciones, colagenopatías, vacunas, anticonceptivos orales, fármacos, citostáticos, trasplante alogénico y enfermedad tumoral. En relación con los tumores, no parece estar en relación con un curso avanzado, en los casos descritos presentaba una remisión objetiva (extremadamente raro como presentación inicial de un tumor). Habitualmente se trata de adenocarcinomas fundamentalmente gástricos, colon, mama y menos con la Leucemia mieloide crónica. El pronóstico cuando existe una causa secundaria es desfavorable. El tratamiento consiste en el aporte de plasma y en la plasmaféresis (sin demora si existe clínica neurológica), siendo la tasa de respuestas en torno al $20 \%$ en los pacientes con cáncer.

\section{I. Gallegos Sancho, M. Reboredo López, R. García Cam- pelo, M. Quindós Varela , L. M Antón Aparicio}

Servicio de Oncología Médica. Complejo Hospitalario Universitario Juan Canalejo. La Coruña

1. Kwaan HC, Gordon LI. Thrombotic Microangiopathy in the Cancer Patient. Acta Haematol 2001; 106: 52-56.

2. Gordon LI, Kwaan HC. Thrombotic Microangiopathy manifesting as Thrombotic Thrombocytopenic Purpura/Hemolytic uremic.

3. Girad P, Tardy B, Page Y, Mosnier JF, Tardy-Poncet B, Bertrand JC. Purpura Thrombotique Thrombocytopénique révélant un cancer: à propos de deux cas. Presse Med 1995; 24: 175-8.

4. Gordon LI, Kwaan HC. Cancer-and Drug-Associated Thrombotic Thrombocytopenic Purpura and Hemolytic uremic Syndrome. Seminars in Hematology 1997; 34: 140-147. 